\section{High prevalence of clustered tuberculosis cases in Peruvian migrants in Florence, Italy}

\author{
Lorenzo Zammarchi, ${ }^{1}$ Enrico Tortoli, ${ }^{2}$ \\ Emanuele Borroni, ${ }^{2}$ Filippo Bartalesi, ${ }^{3}$ \\ Marianne Strohmeyer, ${ }^{1}$ \\ Simonetta Baretti, ${ }^{4}$ \\ Maria Tullia Simonetti, ${ }^{5}$ Carola Liendo, ${ }^{6}$ \\ Maria Grazia Santini, ${ }^{4}$ \\ Gian Maria Rossolini, ${ }^{5}$ Eduardo Gotuzzo, ${ }^{6}$ \\ Alessandro Bartoloni, ${ }^{1,3}$ \\ COHEMI project study group \\ 1'Infectious Diseases Unit, Department \\ of Experimental and Clinical Medicine, \\ University of Florence School of \\ Medicine, Italy; ${ }^{2}$ Emerging Bacterial \\ Pathogens, IRCCS San Raffaele Scientific \\ Institute, Milan, Italy; ${ }^{3 S O D}$ Malattie \\ Infettive e Tropicali, Azienda Ospedaliero \\ Universitaria Careggi, Florence, Italy; ${ }^{4}$ UF \\ Igiene e Sanità Pubblica, ASL 10 Zona \\ Firenze, Florence, Italy; ${ }^{5}$ SOD \\ Microbiologia e Virologia, Centro di \\ Riferimento per i Micobatteri della \\ Regione Toscana, Azienda Ospedaliero \\ Universitaria Careggi, Florence, Italy; \\ 'Instituto de Medicina Tropical Alexander \\ von Humboldt, Universidad Peruana \\ Cayetano Heredia, Lima, Peru
}

\section{Abstract}

Tuberculosis is a leading cause of morbidity for Peruvian migrants in Florence, Italy, where they account for about $20 \%$ of yearly diagnosed cases. A retrospective study on cases notified in Peruvian residents in Florence in the period 2001-2010 was carried out and available Mycobacterium tuberculosis strains were genotyped (MIRU-VNTR-24 and Spoligotyping). One hundred thirty eight cases were retrieved. Genotyping performed in 87 strains revealed that 39 (44.8\%) belonged to 12 clusters. Assuming that in each cluster the transmission of tuberculosis from the index case took place in Florence, a large proportion of cases could be preventable by improving early diagnosis of contagious cases and contact tracing.

\section{Introduction}

As of January 2011, Peruvian migrants represent the largest Latin American community in Italy with 101,711 documented residents accounting for $2.9 \%$ of migrant population, corresponding to about $30 \%$ of Latin Americans in
Italy. ${ }^{1}$ The number of Peruvian citizens in Italy has increased 30 folds since the early nineties. The majority of them are young-adults (70\% aged between 18 and 49 years) and the female gender is slightly prevalent (61\%). The large majority $(70 \%)$ are currently concentrated in few large metropolitan areas namely Milan (33,919 documented residents), Rome $(15,262)$, Turin $(10,872)$, Florence (7834) and Genoa (3277). ${ }^{1}$ In 2009 (the last year with official surveillance data available), 48.3\% (2053) of tuberculosis (TB) cases in Italy were diagnosed in foreign born subjects, with Peruvians accounting for $5 \%$ of them (103 cases). ${ }^{2}$ In the health district of Florence (Azienda Sanitaria Locale, ASL 10), the number of reported TB cases remained almost stable in the last 10 years with around 100-130 cases per year and an incidence of 10-14 cases per 100,000 populations. ${ }^{3}$ Nevertheless, in the same period, the number and the proportion of Peruvian subjects diagnosed with TB in Florence has steadily increased. Starting from 2005, Peruvians represent the foreign community with highest TB burden in Florence, accounting for about $20 \%$ of cases (22 out of 110 in 2009 and 25 out of 133 in 2010). ${ }^{3,4}$

The findings of a retrospective study aiming to understand the epidemiological dynamics of TB transmission in the Peruvian community of Florence are reported here.

\section{Materials and Methods}

Main epidemiological and clinical features of TB cases notified in Peruvian citizens resident in the ASL 10, in the period 2001-2010 were retrospectively collected from the databases of UF Igiene e Sanità Pubblica, ASL 10 Zona Firenze, Florence, Italy and SOD Microbiologia e Virologia, Centro di Riferimento per $i$ Micobatteri della Regione Toscana, Azienda Ospedaliero Universitaria Careggi, Florence, Italy. Incidence of TB in the Peruvian community of Florence was estimated on the basis of number of TB cases notified each year in the ASL 10 (used as numerator) and number of documented Peruvian residents in the province of Florence (used as denominator). ${ }^{5}$ Available Mycobacterium tuberculosis strains isolated were characterized by genotyping analysis with Mycobacterial Interspersed Repetitive Units - Variable Number Tandem Repeat (MIRU-VNTR-24) and Spacer Oligonucleotide Typing (Spoligotyping). ${ }^{6,7}$ Clonally related strains were defined as clustered and assumed to belong to a chain of recent transmission, while patients whose $M$. tuberculosis isolate did not present a shared pattern were assumed to originate from reactivation of a latent tuberculosis infection (LTBI). ${ }^{8}$ As this was a retrospective study on
Correspondence: Alessandro Bartoloni, Largo Brambilla 3, 50134 Florence, Italy.

Tel.Fax: +39.055.794.9431.

E-mail: alessandro.bartoloni@unifi.it

Key words: tuberculosis, immigrants, migrants, cluster, epidemiology, Peru, Italy.

Contributions: LZ, ET, EB, SB, MTS, MGS data collecting and analyzing; $\mathrm{LZ}, \mathrm{AB}$ manuscript writing; MS, FB, EG, GMR, CL manuscript reviewing.

Conflict of interests: the authors declare no potential conflict of interests.

Funding: the study was carried out within the COHEMI (COordinating resources to assess and improve HEalth status of MIgrants from Latin America)-project. This is a three-year collaborative project supported by the European Commission under the Health Cooperation Work Programme of the 7th FRAMEWORK PRO GRAMME (GA-261495) launched in 2011 (http://www.cohemi-project.eu/).

Conference presentation: the preliminary results of this work have been presented at the 8th European Congress on Tropical Medicine and International Health, 10-13 September 2013 Copenhagen, Denmark as poster presentation. An abstract has been published in Tropical Medicine and International Health volume 18 suppl 1 pp 108-231 September 2013 (High prevalence of clustered tuberculosis cases in the Peruvian community in Florence, 2001-2010. P.1.8.005 [B]).

Acknowledgments: authors would thank Elvira Rizzuto (Coordinatore statistico-informatico, Ufficio V - Malattie Infettive e Profilassi Internazionale, Direzione Generale Prevenzione Sanitaria, Ministero della Salute, Italy) and Maria Grazia Pompa (Direttore - Ufficio V Malattie Infettive e Profilassi Internazionale, Direzione Generale Prevenzione Sanitaria, Ministero della Salute, Italy) for providing national data on tuberculosis notification disaggregated for year of notification, sex, region of notification, age, country of birth, age, year of arrival in Italy, anatomical site.

Received for publication: 23 September 2014.

Revision received: 28 October 2014.

Accepted for publication: 29 October 2014

This work is licensed under a Creative Commons Attribution NonCommercial 3.0 License (CC BYNC 3.0).

(C) Copyright L. Zammarchi et al., 2014

Licensee PAGEPress, Italy

Infectious Disease Reports 2014; 6:5646 doi:10.4081/idr.2014.5646

routinely collected surveillance data and on samples collected at the Regional TB reference laboratory, ethical approval was not deemed necessary. 


\section{Results}

One hundred and thirty-eight TB cases were retrieved. Among them, 119 (86\%) were microbiologically confirmed. The number of reported cases steadily increased through the years, from $11(8 \%)$ in 2001-2002 to 47 (34.1\%) in 2009-2010. The estimated incidence rates varied, in the considered period, from 484 and 351 per 100,000 Peruvian residents in Florence. Main epidemiological and clinical features of TB cases are summarized in Table 1.

Eighty-seven $M$. tuberculosis strains were available for genotyping analysis (Figure 1).

MIRU-VNTR-24 analysis showed 48 strains (55.2\%) displaying a non-shared genotype, which were interpreted as originating from LTBI reactivation. Thirty nine (44.8\%) $M$. tuberculosis strains showed a genotype shared at least by another strain, fulfilling the definition of clustered cases. Three clusters included 2 subjects, 3 clusters included 3 subjects, 4 clusters included 4 subjects and 1 cluster included 6 subjects. In the majority of clusters (10 out of 12) the time elapsed between the diagnosis of the first and the last case was shorter than five years. Spoligotyping showed that the lineages circulating in the community were Haarlem ( $\mathrm{n}=31,35.6 \%$, 14 clustered and 17 non-clustered), Latin AmericanMediterranean (LAM, $\mathrm{n}=28,32.1 \%, 12$ clustered and 16 non-clustered), T-specific ( $\mathrm{n}=8$, $9.2 \%, 2$ clustered and 6 non-clustered) Beijing ( $\mathrm{n}=5,5.7 \%, 4$ clustered and 1 non-clustered), Ural ( $n=3,3.4 \%$, all clustered), $S(n=1,1.1 \%)$. Eleven $(12.6 \%, 4$ clustered and 7 non-clustered) previously unreported spoligotypes (orphans) were also present.

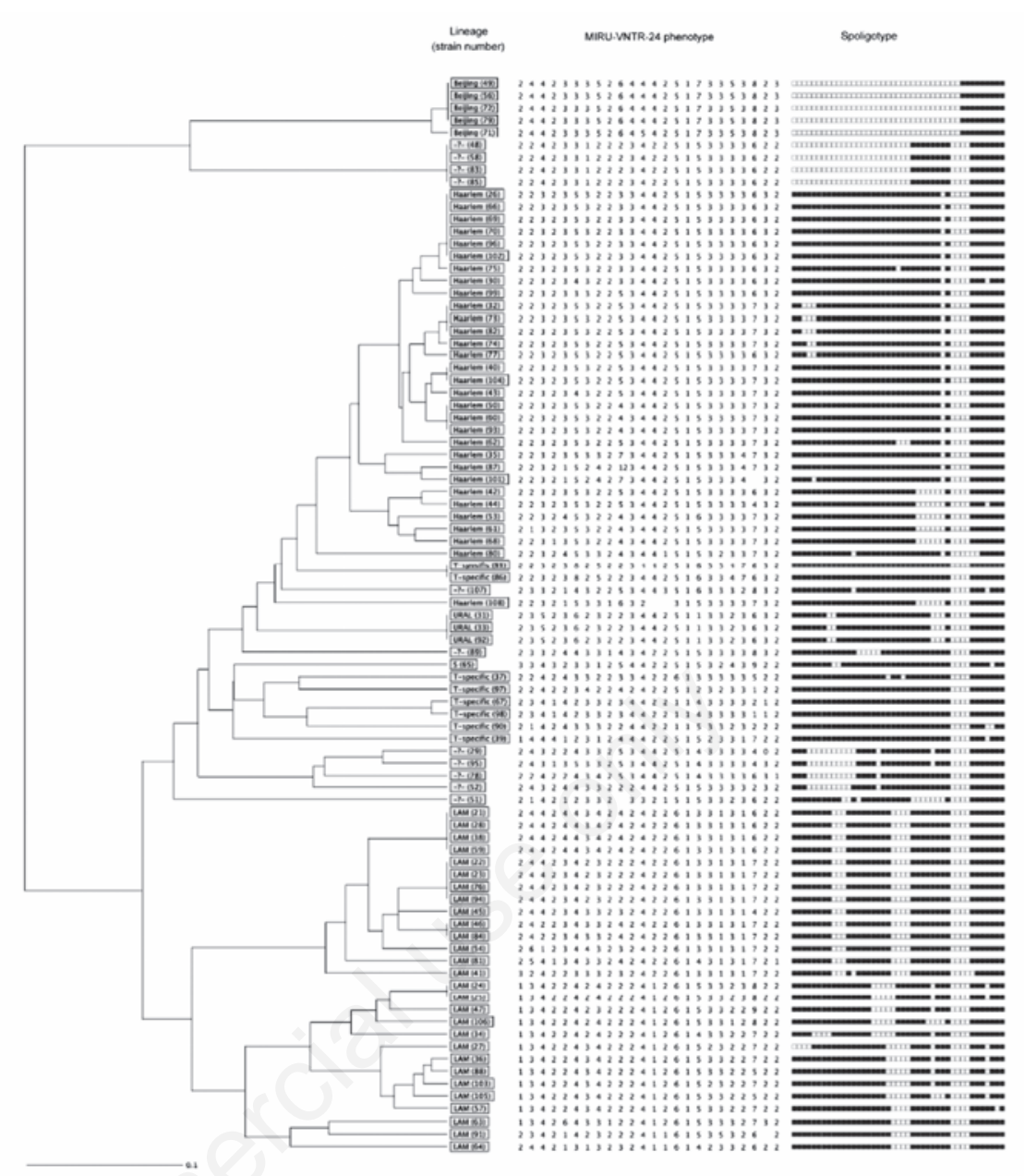

Figure 1. Cluster analysis, on the basis of MIRU-VNTR-24 phenotype and spoligotype, of Mycobacterium tuberculosis strains isolated from 87 Peruvian patients.

Table 1. Clinical, microbiological and epidemiological features of tuberculosis cases notified in Peruvian citizens resident in the Health District of Florence, Italy, in the period 2001-2010.

\begin{tabular}{|c|c|c|c|c|}
\hline & Case & with genotypin & available (87) & All cases* \\
\hline & Total & Clustered (39) & Non clustered (48) & (138) \\
\hline Male/female ratio & 0.6 & 0.8 & 0.4 & 0.7 \\
\hline Age $\leq 15$ years & 9/87 (10.3\%) & 6/39 (15.3\%) & $3 / 48(6.2 \%)$ & 15/138 (10.9\%) \\
\hline Age $16-50$ years & $77 / 87(88.5 \%)$ & $33 / 39(84.6 \%)$ & $44 / 48(91.7 \%)$ & $120 / 138(86.9 \%)$ \\
\hline Age $>50$ years & $1 / 87(1.1 \%)$ & $0 / 39(0 \%)$ & $1 / 48(2.1 \%)$ & $3 / 138(2.2 \%)$ \\
\hline Diagnosis of tuberculosis within 5 years from arrival in Italy & $52 / 83(62.6 \%)$ & $20 / 32(62.5 \%)$ & $32 / 48(66.7 \%)$ & $81 / 116(69.8 \%)$ \\
\hline Diagnostic delay $>60$ days & $32 / 72(44.4 \%)$ & $12 / 32(37.5 \%)$ & $20 / 40(50 \%)$ & $46 / 113(40.7 \%)$ \\
\hline Pulmonary localization only & $68 / 86(79.1 \%)$ & $32 / 38(84.2 \%)$ & $36 / 48(75 \%)$ & 106/136 (77.9\%) \\
\hline Pulmonary and extrapulmonary localization & $5 / 86(5.8 \%)$ & $3 / 38(7.9 \%)$ & $2 / 48(4.2 \%)$ & $8 / 136(5.9 \%)$ \\
\hline Extrapulmonary localization only & $12 / 86(13.9 \%)$ & $3 / 38(7.9 \%)$ & $9 / 48(18.7 \%)$ & $20 / 136(14.7 \%)$ \\
\hline Disseminated tuberculosis & $1 / 86(1.2 \%)$ & $0 / 38(0 \%)$ & $1 / 48(2.1 \%)$ & $2 / 136(1.5 \%)$ \\
\hline Smear positive tuberculosis among subjects with pulmonary localization & $43 / 73(58.9 \%)$ & $19 / 35(54.3 \%)$ & $24 / 38(63.1 \%)$ & $60 / 114(52.6 \%)$ \\
\hline HIV prevalence & $1 / 47(2.1 \%)$ & $0 / 25(0 \%)$ & $1 / 27(3.7 \%)$ & $2 / 73(2.7 \%)$ \\
\hline Pan-susceptible strains & $75 / 84(89.3 \%)$ & $37 / 38(97.4 \%)$ & $38 / 46(82.6 \%)$ & $93 / 113(83.3 \%)$ \\
\hline Multi drug resistant strains & $2 / 84(2.4 \%)$ & $0 / 39(0 \%)$ & $2 / 46(4.3 \%)$ & $9 / 113(8 \%)$ \\
\hline
\end{tabular}

*Includes all tuberculosis cases notified in Peruvian citizens resident in the ASL 10 (Florence, Italy), in the period 2001-2010 regardless of the availability of the genotyping analysis. 


\section{Discussion and Conclusions}

The study shows that TB in the Peruvian community in Florence is a concerning problem considering its high estimated incidence. In 2009, the last year for which final data are available at national level, the estimated incidence of TB in Peruvian born in Italy (117 per $100,000)$ was very similar to the one reported for Peruvian residents in Peru (113 per $100,000)$ and much lower if compared with that found in this study (381 per 100,000). ${ }^{9}$ Supported by the finding of a high rate of clustered cases, we can affirm that the higher TB incidence in Peruvian residents in Florence (3.2 time higher compared with incidence in the country of origin) is related to an ongoing active transmission within the community. In the matter of facts, excluding one case for each cluster that should be considered the index case, the remaining clustered cases are due to recent infections occurred in Florence.

A limitation of this study is that we considered in the analysis only M. tuberculosis strains isolated from Peruvian individuals living in Florence. This greatly limit the possibility of inferring the origin of active TB (new infection $v s$ reactivation) from cluster data. In other word, if a Peruvian patients is not in a cluster with other Peruvian patients, it is not possible to rule out that this patients was not part of a cluster in which the index case was a nonPeruvian patient living in the same area, and thus to infer that TB in that patient was due to reactivation of a remotely acquired infection. Thus the number of patients in cluster should be regarded as a minimum estimate of cases due to recent infection. Peruvian origin has been previously identified as risk factors for TB clustering in a previous Italian study carried out in Milan. ${ }^{10}$ Another study conducted in the late nineties in Milan evidence a 1.6 time higher TB incidence in Peruvian migrants compared with TB incidence in Peru. ${ }^{10}$ Considering data obtained in the studies carried out in Milan and in Florence, Peruvian migrant communities of both cities emerge as a vulnerable group which may benefit from targeted intervention and TB control activities. Strengthening of preventive intervention, such as early diagnosis of active TB cases and contact tracing procedures, should be prioritized for this population. An easy access to TB services for the members of this communities should be assured, regardless of legal status of subjects. Collaboration between different partners such as public health services, primary care physicians, tertiary hospitals and non-for profit sector is warranted to achieve a timely diagnosis of TB and to ensure case holding of affected subjects. Socially and culturally acceptable education programs on TB with a multidisciplinary approach should be reinforced.

\section{The COHEMI project study group}

Authors would also thank the COHEMI project study group that includes: Maurizio Bonati (a), Chiara Pandolfini (a), Francesca Severino (a), Valeria Confalonieri (a), Gianni Tognoni (a), Zeno Bisoffi (b), Dora Buonfrate (b), Andrea Angheben (b), Marco Albonico (b,c), Alessandro Bartoloni (d), Marianne Strohmeyer (d), Lorenzo Zammarchi (d), Filippo Bartalesi (e), Jose Muñoz (f), Ana Requena-Mendez (f), Maria Roura (f), Laia Ventura (f), Robert Pool (g), Christopher Pell (g), Anita Hardon (g), Peter Chiodini (j), Juan Moreira (k), Mariella Anselmi (k), Roberto Sempértegui (k), Eduardo Gotuzzo (l), Maria Alejandra Mena (l), Carola Liendo (l), Héctor H. Garcia (m), Javier Bustos (m), Saul Santivañez (m), Faustino Torrico (n), Daniel Lozano (n), Teresa Hinojosa Cabrera (0), Javier Ochoa Morón (0), Ignacio Abapori Cuellar (o), Jaime Amorós Suarez (0), Guido Chumiray Rojas (o), Alessandra Nicoletti (p), Elisa Bruno (p).

(a) Department of Public Health, Laboratory for Mother and Child Health, 'Mario Negri' Pharmacological Research Institute, Milan, Italy. b) Centre for Tropical Diseases, Sacro Cuore-Don Calabria Hospital, Negrar, Verona, Italy. c) Ivo de Carneri Foundation, Milano, Italy. d) Infectious Disease Unit, Department of Experimental \& Clinical Medicine, University of Florence School of Medicine, Florence, Italy. e) SOD Malattie Infettive e Tropicali, Azienda Ospedaliero-Universitaria Careggi, Florence, Italy. f) Servicio de Medicina Tropical y Salud Internacional, Centre de Recerca en Salut Internacional de Barcelona, Hospital Clínic-Universitat de Barcelona, Barcelona, Spain. g) Centre for Social Science and Global Health, University of Amsterdam, The Netherlands. j) Hospital for Tropical Diseases and London School of Hygiene \& Tropical Medicine, London, United Kingdom. k) Centre for Community Epidemiology and Tropical Medicine, Esmeraldas, Ecuador. l) Instituto de Medicina Tropical Alexander von Humboldt, Universidad Cayetano Heredia, Lima, Peru. m) Cysticercosis Unit, Instituto de Ciencias Neurologicas, Department of Microbiology, Universidad Peruana Cayetano Heredia, Lima, Peru. n) Colectivo de Estudios Aplicados y Desarrollo Social, Cochabamba, Bolivia. 0) Taller de Educacion y Comunicacion TEKOGUARANÌ, Camiri, Bolivia. p) Dipartimento G.F. Ingrassia Sezione di Neuroscienze, Università di Catania, Catania, Italy.

\section{References}

1. Direzione Generale dell'Immigrazione e delle Politiche di Integrazione, Ministero del Lavoro e delle Politiche Sociali. La Comunità Peruviana in Italia. Rapporto annuale sulla presenza degli immigrati 2012. Available from: http://www.integrazionemigranti.gov.it/archiviodocumenti/a re e-di-origine/D o c uments/ Rapporto\%20Peru\%20def.pdf

2. Ministero del Lavoro e delle Politiche Sociali, Direzione Generale Prevenzione Sanitaria, Ufficio V - Malattie Infettive e Profilassi Internazionale. Data on tuberculosis notification disaggregated for year of notification, sex, region of notification, age, country of birth, age, year of arrival in Italy, anatomical site. Available from: http://www.salute.gov.it/portale/temi/p2_6.j sp?lingua $=$ italiano\&id $=831 \&$ are $=$ Malatt ie\%20infettive\&menu=altremalattie

3. Barchielli A, Baretti S, Baroncini 0, et al. La tubercolosi... attenzione sì, allarmismo no! (report 2010). Available from: http://www.asf.toscana.it/images/stories/e pidemiologia/epi_tbc_report_2011.pdf.

4. Barchielli A, Baretti S, Baroncini 0, et al. La tubercolosi nella ASL 10 (report 2009). Available from: http://www.asf.toscana.it/ images/stories/epidemiologia/epi_tbc_rep ort_2009.pdf

5. Istituto Nazionale di Statistica. Bilancio demografico e popolazione residente straniera al 31 dicembre per sesso e cittadinanza. Available from: http//demo.istat.it/ archivio.html

6. Kamerbeek J, Schouls L, Kolk A, et al. Simultaneous detection and strain differentiation of Mycobacterium tuberculosis for diagnosis and epidemiology. J Clin Microbiol 1997;35:907-14.

7. Supply P, Lesjean S, Savine E, et al. Automated high-throughput genotyping for study of global epidemiology of Mycobacterium tuberculosis based on mycobacterial interspersed repetitive units. J Clin Microbiol 2001;39:3563-71.

8. Franzetti F, Codecasa L, Matteelli A, et al. Genotyping analyses of tuberculosis transmission among immigrant residents in Italy. Clin Microbiol Infect 2010;16:114954.

9. World Health Organization. Global tuberculosis control 2011. Available from: http://whqlibdoc.who.int/publications/2011 /9789241564380_eng.pdf

10. Codecasa LR, Porretta AD, Gori A, et al. Tuberculosis among immigrants from developing countries in the province of Milan, 1993-1996. Int J Tuberc Lung Dis 1999;3:589-95 